\title{
EFFECT OF CONDUCTING AND RADIATING GASES ON THE GROWTH AND DECAY OF WEAK MAGNETOGASDYNAMIC WAVES
}

\author{
A. RAI \\ Department of Malhcmatics, NERIST, Nirjuli-791 109, Itanagar, A.P., India
}

AND S.P.S. SISODIA

Department of Computer Science, NERIST, Itanagar, A.P., Nirjuli-791 109, India

(Received June 26, 1991; revised version April 8, 1992)

\begin{abstract}
Singular surface theory is applicd to study the growth and decay properties of weak magnetogasdynamic discontinuities under the influences of thermal conduction and the time-dependent radiation field interacting with magnetogasdynamic field with finite electrical conductivity. It is shown that the time-dependent radiation field gives rise to a radiation induced weak wave which is always damped and the magnetogasdynamic waves which break at the wave front if the initial discontinuity is sufficiently strong. The wave front curvature effects on the weak magnetogasdynamic waves are also discussed.
\end{abstract}

PACS numbers: $47.40 . \mathrm{Nm}$

\section{Introduction}

With the advancement of space technology the propagation of waves in gaseous media at very high temperature and at low density becomes an interesting problem in determining the flow field. In such situations we can not justify the neglect of the effects of thermal radiation, thermal conduction and electromagnetism. These effects require a study of a complicated interaction among the radiation field, the electromagnetic field and the gasdynamic field simultaneously. The inclusion of radiation makes the fundamental gasdynamic relationships a complex. set of nonlinear integrodifferential equations when accounting for the frequency dependence of the radiation field. Such a situation arises when a solar wind of fully ionized plasma interacts with a plasma column of Earth's atmosphere and compresses it. As a result of interaction, weak wave characteristics emerge and with changing inclinations, they intersect to form a shock wave. 
Since, at high temperatures, a gas is likely to be fully or partially ionized, the electromagnetic effects may play a decisive role in the determination of the flow field. One is thus led to study the interaction of radiative and electromagnetic effects arising in the problems of solar photosphere, rocket re-entry and elsewhere. Also at high temperatures, it is more realistic to consider the contribution of the thermal conduction effects along with the radiation and the magnetic field with finite electrical conductivity in a gaseous flow field. These ideas give some insight into interactions of various mechanisms participating in the wave propagation.

Thomas [1] introduced the singular surface theory to study the propagation of weak waves in an ideal gas flow. Several authors [2-5] further generalized and developed the singular surface theory to cover up some complicated cases of real gases. Nariboli and Secrest [6] extended the analysis of Thomas to magnetogasdynamic flows with finite electrical conductivity. During the last two decades several investigators [7-10] studied thermal radiation effects on the propagation of small disturbances in gas flows under steady state conditions. The growth and decay properties of the weak waves in radiation gasdynamics have been discussed by Srinivasan and Ram [11], Ram [12], Ram and Pandey [13]. Shankar and Prasad [14] have extended the paper [11] taking into account the unsteady motion ahead of the wave surface. In the above studies, the approximation to the radiative heat transfer equation is too strong and the gas is assumed optically thin or thick. Most of the authors [7-15] have neglected the time dependence in the radiative heat transfer equations. The neglect of the time derivative of the radiation field amounts to the suppression of one mode of wave propagation excited by radiation and, therefore, the exact behaviour of waves in radiation gasdynamics is not fully understood.

After incorporating the idea of the time-dependent radiation field, the governing equations clearly show the existence of radiation induced waves. The object of the present work is to obtain the growth equation which will govern the growth and decay of weak magnetogasdynamic waves taking into account the effects of thermal conduction and the time-dependent radiation field interacting with the magnetogasdynamic field with finite electrical conductivity. The study of the effect of thermal radiation is based on the differential approximation [16] of the radiative heat transfer equation. The influences of the thermal radiation, thermal conduction, the magnetic field and that of the initial wave front curvature are discussed on the nonlinear breaking of modified magnetogasdynamic (MGD) waves.

\section{Basic equations}

The set fo nonlinear differential equations governing three-dimensional flow of a radiation magnetogasdynamics with finite electrical conductivity $\sigma$ is

$$
\begin{aligned}
& \frac{\partial \rho}{\partial t}+u_{i} \rho_{, i}+\rho u_{i, i}=0 \\
& \rho \frac{\partial u_{i}}{\partial t}+\rho u_{j} u_{i, j}+p_{, i}+p_{, i}^{\mathrm{R}}+\mu_{0} I_{j} H_{j, i}-\mu_{0} H_{j} H_{i, j}=0,
\end{aligned}
$$




$$
\begin{aligned}
& \frac{\partial E}{\partial t}+\left[u_{i}\left(E+p+p^{\mathrm{R}}\right)+q_{i}^{\mathrm{R}}-K T_{, i}\right]_{, i}=J^{2} / \sigma, \\
& \frac{\partial H_{i}}{\partial t}+u_{j} H_{i, j}-H_{j} u_{i, j}+H_{i} u_{j, j}-\left(\sigma \mu_{0}\right)^{-1} H_{i, j j}=0, \\
& J_{i}=\varepsilon_{i j k} H_{k, j}, \quad J^{2}=J_{i} J_{i}, \quad p=\rho R T,
\end{aligned}
$$

where $u_{i}, H_{i}, p, \rho, J, R$ and $T$ represent the components of flow velocity, the components of the magnetic field, the gas pressure, the gas density, the electrical current density, the universal gas constant and the absolute gas temperature, respectively. $E$ is the total energy per unit volume, $p^{\mathrm{R}}$ is the radiation pressure and $q_{i}^{\mathrm{R}}$ is the radiative heat flux vector. The constants $\gamma, K$ and $\mu_{0}$ represent the heat exponent of the gas, the coefficient of the thermal conduction and the magnetic permeability, respectively and $t$ denotes the time. A comma followed by an index $i$ denotes the partial differentiation with respect to the spatial coordinate $x^{i}$.

Under the differential approximation the equations of radiative heat transfer may be written as the pair of equations [16]:

$$
\begin{aligned}
& \frac{\partial E^{\mathrm{R}}}{\partial t}+q_{i, i}^{\mathrm{R}}+\alpha\left(c E^{\mathrm{R}}-4 a_{\mathrm{R}} T^{4}\right)=0, \\
& \frac{1}{c} \frac{\partial q_{i}^{\mathrm{R}}}{\partial t}+\frac{1}{3} c E_{, i}^{\mathrm{R}}+\alpha q_{i}^{\mathrm{R}}=0,
\end{aligned}
$$

where $E^{\mathrm{R}}=3 p^{\mathrm{R}}$ is the radiative energy density per unit volume, $\alpha$ is a grey absorption coefficient, $c$ is the speed of light and $a_{\mathrm{R}}$ is the Stefan-Boltzmann constant.

The energy equation (3) can be simplified in the form

$$
\begin{aligned}
& \left(\frac{\partial p}{\partial t}+u_{i} p_{, i}\right)+3(\gamma-1)\left(\frac{\partial p^{\mathrm{R}}}{\partial t}+u_{i} p_{, i}^{\mathrm{R}}\right)+\left[\gamma p+4(\gamma-1) p^{\mathrm{R}}\right] u_{i, i}+(\gamma-1) \\
& \times \mu_{0} u_{i}\left(H_{j} H_{i, j}-H_{j} H_{j, i}\right)+(\gamma-1) q_{i, i}^{\mathrm{R}}-(\gamma-1) K T_{, i i}=(\gamma-1) J^{2} / \sigma .
\end{aligned}
$$

We assume that there exist a surface of discontinuity $S(t)$ in the flow field, which is such that the magnetic field with the first derivatives and all other flow variables themselves are continous across it, but the second derivatives of the magnetic field and the first derivatives of olher variables are discontinuous or undergo finite jumps. Such a jump discontinuity is defined as a weak MGD discontinuity or a weak MGD wave. The geometric and kinematic compatibility conditions derived in $[17]$ reduce to

$$
\begin{aligned}
& {\left[Z_{, i}\right]=B n_{i}, \quad\left[\frac{\partial Z}{\partial t}\right]=-B G} \\
& {\left[Z_{, i j}\right]=\bar{B} n_{i} n_{j}+g^{\alpha \beta} B_{, \alpha}\left(n_{i} x_{j, \beta}+n_{j} x_{i, \beta}\right)-B g^{\alpha \beta} g^{\mu \nu} b_{\alpha \mu} x_{i, \beta} x_{j, \nu}} \\
& {\left[\frac{\partial^{2} Z}{\partial x_{i} \partial t}\right]=\left(-G \bar{B}+\frac{\delta B}{\delta t}\right) n_{i}-g^{\alpha \beta}(B G)_{, \alpha} x_{i, \beta},} \\
& {\left[\frac{\partial^{2} Z}{\partial t^{2}}\right]=G^{2} \bar{B}-2 G \frac{\delta B}{\delta t}-B \frac{\delta G}{\delta t}}
\end{aligned}
$$


where $Z$ may be any of the flow variables $p, \rho, u_{i}, H_{i}, q_{i}^{\mathrm{R}}$, and $p^{\mathrm{R}}$ etc. and the scalar quantity $B=\left[Z_{, i}\right] n_{i}, \bar{B}=\left[Z_{, i j}\right] n_{i} n_{j}$ are defined over $S(l), G$ is the normal speed of the propagation of the surface $S(l)$ and $n_{i}$ are the components of the unit vector normal to it.

Since the law of conservation of encrgy holds across a weak MGD discontinuity surface, the energy equation (3) implies that

$$
K\left[T_{, i}\right] n_{i}=\left[q_{i}^{\mathrm{R}}\right] n_{i}=0 .
$$

Forming jumps across $S(l)$ in Eqs. (1), (2), (6), (7) and using (9), we get

$$
\begin{aligned}
& \left(u_{0 n}-G\right) \zeta+\rho_{0} \lambda_{i} n_{i}=0, \\
& \rho_{0}\left(u_{0 n}-G\right) \lambda_{i}+\xi n_{i}+0 n_{i}=0, \\
& -3 G 0+\varepsilon_{i} n_{i}=0, \\
& -G \varepsilon_{i}+c^{2} 0 n_{i}=0
\end{aligned}
$$

where

$$
\begin{aligned}
& \lambda_{i}=\left[u_{i, j}\right] n_{j}, \quad \xi=\left[p_{, i}\right] n_{i}, \quad \zeta=\left[\rho_{i}\right] n_{i}, \\
& \theta=\left[p_{, i}^{\mathrm{R}}\right] n_{i}, \quad \varepsilon_{i}=\left[q_{i, j}^{\mathrm{R}}\right] n_{j}, \quad u_{0 n}=u_{0 i} n_{i}
\end{aligned}
$$

and the suffix " 0 " denotes the evaluation just ahead of the propagating wave surface.

Differentiating Eq. (5c) partially with respect to $x_{i}$, using (10) and applying the compatibility conditions (9), we get

$$
\rho_{0} \xi=p_{0} \zeta \text {. }
$$

Equations (11)-(15) constitute a set of nine equations with nine unknown quantitics $\lambda_{i}, \varepsilon_{i}, \xi, \zeta$ and $\theta$. The system has a nontrivial solution, if $G=u_{0 n}+a_{01}$ or $G= \pm c / \sqrt{3}$, where $a_{01}=\sqrt{p_{0} / \rho_{0}}$ is the isothermal speed of sound. For advancing wave surface, we shall take $G$ to be positive. We thus find that there are two types of weak discontinuity surfaces, one of which propagates with an isothermal speed of sound relative to the gas flow and is defined as a gasdynamic weak wave and the other one propagates with the velocity $c / \sqrt{3}$ and is defined as radiation induced weak wave.

Taking jump in Eq. (4) again and using (9) we get

$$
\bar{\eta}_{i}=\sigma \mu_{0}\left(H_{0 i} \lambda_{n}-I_{0 n} \lambda_{i}\right),
$$

where

$$
\bar{\eta}_{i}=\left[H_{i, j k}\right] n_{j} n_{k} .
$$

From the relations (11) and (16) we obtain

$$
\lambda_{i}=\psi n_{i}, \quad \zeta=\left(\rho_{0} / a_{01}\right) \psi, \quad \bar{\eta}_{i}=\sigma \mu_{0} I I_{0}\left(l_{i}-l_{n} n_{i}\right) \psi,
$$

where $\psi$ may be defined as the amplitude of a weak MGD wave. Ifcre $l_{i}$ represents the components of the unit vector in the direction of magnetic field. 


\section{Behaviour of radiation induced wave}

When the first-order discontinuilies propagate along the radiation induced wave $S(t)$, Eqs. (11)-(15), using $G=c / \sqrt{3}$ yield

$$
\begin{aligned}
& \theta^{\mathrm{R}}=\varepsilon^{\mathrm{R}} / c \sqrt{3}, \\
& \lambda^{\mathrm{R}}=\varepsilon^{\mathrm{R}}\left[3 \rho_{0} c^{2}\left(1 / 3-a_{01}^{2} / c^{2}\right)\right]^{-1}, \\
& \zeta^{\mathrm{R}}=\varepsilon^{\mathrm{R}}\left[\sqrt{3} c^{3}\left(1 / 3-a_{01}^{2} / c^{2}\right)\right]^{-1}, \\
& \xi^{\mathrm{R}}=a_{01}^{2} \varepsilon^{\mathrm{R}}\left[\sqrt{3} c^{3}\left(1 / 3-a_{01}^{2} / c^{2}\right)\right]^{-1},
\end{aligned}
$$

where $\lambda^{\mathrm{R}}=\lambda_{i}^{\mathrm{R}} n_{i}, \varepsilon^{\mathrm{R}}=\varepsilon_{i}^{\mathrm{R}} n_{i}$ and the supcrscript $\mathrm{R}$ denotes a jump discontinuity associated with a radiation induced wave.

When Eq. (6) with the help of Eq. (7) differentiated partially with respect to $t$ and the jumps are taken across $S(t)$, we find, using $G=c / \sqrt{3}$ and compatibility conditions [9], that

$$
\frac{\partial \varepsilon^{\mathrm{R}}}{\partial t}+c\left(\alpha_{0}-1 / \sqrt{3} \Omega\right) \varepsilon^{\mathrm{R}}=0 .
$$

Equation (22) is the differential equation governing the growth and decay of a radiation induced wave. The mean curvature at any point of the wave surface has the representation [18]:

$$
\Omega=\left(\Omega_{0}-K_{0} G t\right) /\left(1-2 \Omega_{0} G t+K_{0} G^{2} \iota^{2}\right),
$$

where $\Omega_{0}$ and $K_{0}$ are, respectively, the mean and Gaussian curvatures of $S(t)$ at $t=0$ and $G$ is the constant speed of propagation of the wave surface.

Equation (22) using (23) can be integrated and yields

$$
\varepsilon^{\mathrm{R}}=\varepsilon_{0}^{\mathrm{R}} \exp \left(-\alpha_{0} c t\right) \exp \left(c / \sqrt{3} \int_{0}^{t} \Omega \mathrm{d} t\right),
$$

where $\varepsilon_{0}^{\mathrm{R}}$ is the value of $\varepsilon^{\mathrm{R}}$ at $\iota=0$.

It is obvious from Eq. (24) that $\varepsilon^{R} \rightarrow 0$ as $t \rightarrow \infty$, i.e. the radiation induced waves are damped and the formation of a front carrying discontinuities in the flow variables, is not possible from a continuous flow. It means that the jump discontinuities in the flow variables decay very fast with time $l$. Since $c$ is very large, it follows from (19), (20) and (21) that any disturbance caused by a radiation induced weak wave has a negligibly small influence on the gasdynamic flow field. Thus there is no loss in conșidering that the undisturbed side of the wave is in constant state at rest.

\section{Behaviour of a MGD weak wave}

Now we shall study the bchaviour of a weak MGD discontinuity into a medium which is in a constant state at rest. The jump discontinuities $\xi, \zeta, \lambda$ and $\theta, \varepsilon$ are connected by the relation

$$
\xi=a_{01}^{2} \zeta=a_{01} \rho_{0} \lambda,
$$




$$
\theta=\varepsilon_{i} n_{i}=0
$$

When Eqs. (1) and (2) are differentiated partially with respect to $x_{k}$ and jumps are taken across $S(\mathrm{t})$, we find, using compatibility conditions of second order [9]:

$$
\begin{aligned}
& -a_{01}^{2} \bar{\zeta}+\rho_{0} \frac{\partial \psi}{\partial t}+\rho_{0} a_{01} \bar{\lambda}_{i} n_{i}-2 \rho_{0} a_{01} \Omega \psi+2 \rho_{0} \psi^{2}=0 \\
& -\rho_{0} a_{01} \bar{\lambda}_{i} n_{i}+\rho_{0} \frac{\partial \psi}{\partial t}+\bar{\xi}+\bar{\theta}+\sigma \mu_{0} \rho_{0} b_{0}^{2}\left(1-l_{n}^{2}\right) \psi=0
\end{aligned}
$$

where

$$
\begin{aligned}
& \bar{\lambda}_{i}=\left[u_{i, j k}\right] n_{j} n_{k}, \quad \bar{\zeta}=\left[\rho_{, j k}\right] n_{j} n_{k}, \quad \bar{\xi}=\left[p_{, j k}\right] n_{j} n_{k}, \\
& \bar{\theta}=\left[p_{, j k}^{\mathrm{R}}\right] n_{j} n_{k} \quad \text { and } \quad \mu_{0} H_{0}^{2}=\rho_{0} b_{0}^{2} .
\end{aligned}
$$

Differentiating Eq. (5c) twice partially with respect to $x_{i}$ and applying compatibility conditions [9], we find a relation of the form

$$
\bar{\xi}=\rho_{0} R \bar{\phi}+a_{01}^{2} \bar{\zeta}
$$

where

$$
\bar{\phi}=\left[T_{, i j}\right] n_{i} n_{j} .
$$

Applying the compatibility conditions (9) and using (26), (29) and (17), we find that

$$
-\rho a_{01}^{2} \psi+\left[\gamma p_{0}+4(\gamma-1) p_{0} R_{p 0}\right] \psi=(\gamma-1) / \rho_{0} R\left(\bar{\xi}-a_{01}^{2} \bar{\zeta}\right) .
$$

Differentiating (6) and (7) partially with respect to $x_{k}$, taking the jumps and applying the compatibility conditions [9], we find that $\bar{\theta}=0$. Now eliminating $\bar{\lambda}_{i} n_{i}, \bar{\zeta}$ and $\bar{\xi}$ from (27), (28) and (30) and using (25), we obtain a growth equation for $\psi$ in the form

$$
\frac{\partial \psi}{\partial t}+\left\{(1 / 2)\left[p_{0} R\left(1+4 R_{p 0}\right) / K+\mu_{0} \sigma b_{0}^{2}\left(1-l_{n}^{2}\right)\right]-a_{0 I} \Omega\right\} \psi+\psi^{2}=0,
$$

where $\Omega$ is the mean curvature of the propagating wave surface.

If $\beta$ is the angle between the direction of the magnetic field and that of the normal to wave front so that $l_{i} n_{i}=\cos \beta$, the effective term is $b_{0}^{2} \sin ^{2} \beta$, which is the tangential component. IIence, we have

$$
\frac{\partial \psi}{\partial t}+\left\{(1 / 2)\left[p_{0} R\left(1+4 R_{p 0}\right) / K+\sigma \mu_{0} b_{0}^{2} \sin ^{2} \beta\right]-a_{01} \Omega\right\} \psi+\psi^{2}=0,
$$

Since $S(l)$ represents a moving singular surface of a weak MGD wave at any time $l$ and $G$ is a constant for a uniform medium at the rest ahead of the wave, we have

$$
\frac{\partial n_{i}}{\partial t}=0 \text {. }
$$

Therefore, $S(l)$ will represent a family of parallel surfaces. If $r$ denotes the distance of $S(t)$ from $S(0)$ measured along the normal to $S(0)$, we can write $r=G t$, where

$$
\frac{\partial r}{\partial t}=G
$$


Thus we can write (32) in the form

$$
\frac{\mathrm{d} \psi}{\mathrm{d} t}+\left[Q_{0}-a_{01} \Omega(t)\right] \psi+\psi^{2}=0,
$$

where

$$
Q_{0}=(1 / 2)\left[p_{0} R\left(1+4 R_{p 0}\right) / K+\sigma \mu_{0} b_{0}^{2} \sin ^{2} \beta\right] .
$$

Equation (33) is the required differential equation governing the growth and decay of weak MGD waves in a radiation induced flow field. Equation (33) using (23) can be integrated and then it yields

$$
\psi=\frac{\psi_{0} \exp \left(-Q_{0} t\right)\left(1-2 \Omega_{0} a_{01} t+K_{0} a_{01}^{2} t^{2}\right)^{-1 / 2}}{1+\psi_{0} \int_{0}^{t}\left[\exp \left(-Q_{0} t^{\prime}\right)\left(1-2 \Omega_{0} a_{01} t^{\prime}+K_{0} a_{01}^{2} t^{2}\right] \mathrm{d} t^{\prime}\right.}
$$

where $\psi_{0}$ is the value of $\psi$ at $t=0$.

To discuss the physical aspects of Eq. (34), we will consider the two cases of plane and cylindrical waves, respectively.

\subsection{Plane waves}

For a plane wave front $\Omega_{0}=0=K_{0}$, Eq. (34) yields

$$
\psi=\psi_{0} \exp \left(-Q_{0} t\right) /\left\{1+\left(\psi_{0} / \psi_{\mathrm{c}}\right)\left[1-\exp \left(-Q_{0} t\right)\right]\right\},
$$

where

$$
\psi_{\mathrm{c}}=Q_{0} .
$$

Equation (35) shows that if $\psi_{0}>0$ (i.e. an expansion wave front) and $Q_{0}>0$, then the denominator of (35) remains positive and $\psi=0$ as $t \rightarrow \infty$. It means that the wave decays. Also if $\psi_{0}<0$ (i.e. a compressive wave front) and if it has the magnitude less than $\psi_{\mathrm{c}}$, then the denominator of (35) remains positive and finite as $t \rightarrow \infty$, while the numerator tends to zero, i.e. $\psi \rightarrow 0$ as $t \rightarrow \infty$. It means that the compressive wave decays and damps out ultimately.

Further, if $\psi_{0}=-\psi_{c}$ then $\psi=\psi_{0}$, i.e. the wave propagates with the initial discontinuity without any growth or decay. But if $\psi_{0}<0$ and has a magnitude greater than $\psi_{\mathrm{c}}$, then $\psi$ increases without any limit for a finite time $t_{\mathrm{c}}$ given by

$$
t_{c}=\left(1 / Q_{0}\right) \log \left[1+\left(\psi_{\mathrm{c}} / \psi_{0}\right)\right]^{-1} .
$$

Thus, when $\psi \rightarrow 0$ as $t \rightarrow t_{c}$, a compressive wave discontinuity will break down and a shock type discontinuity will appear spontaneously. The underlying fact is that as a consequence of exceedingly large gradients the flow parameters themselves become discontinuous and the flow cannot be maintained without the presence of a shock wave. From Eq. (36) it follows that $\partial \iota_{c} / \partial Q_{0}>0$, i.e. an increase in $Q_{0}$ causes an increase in the time $t_{\mathrm{c}}$ of the breaking on the wave front, so we can conclude that the effects of thermal radiation through $R_{p 0}$ and the magnetic field with finite electrical conductivity $\sigma$ are to increase the time of breaking, while the thermal conduction effect through $K$ is to decrease the time of breaking. Thus a shock formation is either delayed or disallowed due to thermal radiation and magnetic field effects. On the other hand, the thermal conduction effect helps in the breakdown of a weak MGD wave into a shock wave. 
When $Q_{0}<0$, it follows from Eq. (35) that if $\psi_{0}>0$ when $\psi \rightarrow\left|\psi_{\mathrm{c}}\right|$ as $t \rightarrow \infty$, i.e. breaking is not possible at the wave fronts, the wave takes a stable form. But if $\psi_{0}<0$ and $Q_{0}<0$, then we have

$$
\bar{t}_{\mathrm{c}}=1 /\left|Q_{0}\right| \log \left(1+\left|\psi_{\mathrm{c}}\right| /\left|\psi_{\mathrm{o}}\right|\right)
$$

for the nonlinear breaking of the wave front. Thus in this case a shock-type discontinuity will develop at time $\bar{t}$. Further in this case $\partial \vec{t}_{\mathrm{c}} / \partial\left|Q_{0}\right|<0$, i.e. $\bar{t}_{\mathrm{c}}$ is a decreasing function of $\left|Q_{0}\right|$. Thus it is obvious that the thermal radiation and magnetic field with the finite electrical conductivity with $Q_{0}<0$ are to decrease the time of breaking of the weak waves, while the effect of thermal conduction with $Q_{0}<0$ is to increase the time of breaking of weak waves into shock waves.

\subsection{Cylindrical waves}

If the outgoing discontinuity surface is a cylinder of radius $R_{0}$ at $t=t_{0}$, then at any time $t>t_{0}$ the radius of the cylinder is given by $R=R_{0}+a_{01} t$, in this case $\Omega_{0}=-1 / 2 R_{0}$ and $K_{0}=0$ and Eq. (35) reduces to the form

$$
\psi=\frac{\psi_{0}\left(R_{0} / R\right)^{1 / 2} \exp \left[-Q_{0}\left(R-R_{0}\right) / a_{01}\right]}{1+\left(\psi_{0} / \psi_{\mathrm{c}}^{*}\right)\left[1-\operatorname{er}\left[\mathrm{c}\left(Q_{0} R / a_{01}\right)^{1 / 2} / \operatorname{crfc}\left(Q_{0} R_{0} / a_{01}\right)^{1 / 2}\right]\right.},
$$

where

$$
\psi_{\mathrm{c}}^{*}=\frac{\left(Q_{0} a_{01} / \pi R_{0}\right)^{1 / 2} \exp \left(-Q_{0} R_{0} / a_{01}\right)}{\operatorname{erfc}\left(Q_{0} R_{0} / a_{01}\right)^{1 / 2}}
$$

is a positive critical value of the initial discontinuity in the sense discussed below and

$$
\operatorname{erfc}(x)=(2 / \sqrt{\pi}) \int_{x}^{\infty} \exp \left(-\iota^{2}\right) \mathrm{d} t
$$

is the complementary error function. If $Q>0$, the term inside the curly bracket in the denominator of (37) increases from 0 to 1 as $R$ increases from $R$ to $\infty$. Thus, if $\psi_{0}>0$ or $\psi_{0}<0$ and $\left|\psi_{0}\right|=\psi_{\mathrm{c}}^{*}$, we have from (37) that $\psi \rightarrow 0$ as $R \rightarrow \infty$, i.e. the wave decays and damps out, finally. And if $\psi_{0}<0$ and $\left|\psi_{0}\right|=\psi_{\mathrm{c}}^{*}$ at time $t$, the discontinuity $|\psi|$ is given by

$$
|\psi|=\frac{2\left(Q_{0} a_{01} / \pi R_{0}\right)^{1 / 2} \exp \left(-Q_{0} R_{0} / a_{01}\right)}{\operatorname{erfc}\left(Q_{0} R_{0} / a_{01}\right)^{1 / 2}},
$$

which shows that a compressive wave will neither terminate into a shock wave nor completely damp out. By l'IIospital's rule, we get from (39) that $\psi \rightarrow Q_{0}$ as $R \rightarrow \infty$, i.e. the compressive wave will assume a stable form. Now if $\psi_{0}<0$ and $\left|\psi_{0}\right|>\psi_{\mathrm{c}}^{*}$, there exists a non-zero finite time $t_{\mathrm{c}}^{*}$ given by

$$
\operatorname{erfc}\left(Q_{0} t_{\mathrm{c}}^{*}+Q_{0} R_{0} / a_{01}\right)^{1 / 2}=\left(1-\psi_{\mathrm{c}}^{*} / \psi_{0}\right) \operatorname{erfc}\left(Q_{0} R_{0} / a_{01}\right)^{1 / 2},
$$

such that $|\psi| \rightarrow \infty$ as $t \rightarrow t_{c}^{*}$. This shows that the nonlinear breaking will occur on the wave front in a finite time $l_{\mathrm{c}}^{*}$, i.e. a compressive wave discontinuity will break down and a shock type discontinuity will appear. We define $\psi_{\mathrm{c}}^{*}$ as the critical value of the initial discontinuity $\psi_{0}$ for cylindrical waves. Using the inequality $\operatorname{erfc}(x)<\exp \left(-x^{2}\right) /(x \sqrt{\pi})$, it follows from (38) that $\psi_{\mathrm{c}}^{*}>Q_{0}$ or $\psi_{\mathrm{c}}^{*}>\psi_{\mathrm{c}}$, i.e. the critical value of the initial discontinuity for a cylindrical wave is always greater than 
that of plane wave. Also we have from (40) that $\partial t_{c}^{*} / \partial Q_{0}>0$ and $\partial l_{c}^{*} / \partial R_{0}<0$, which imply that an increase in $Q_{0}$ or an increase in the initial curvature will increase the time breaking of the wave front. Now for $Q_{0}<0$, the growth and decay phenomenon is again similar to that of plane waves, i.e. if $\psi_{0}>0$, then $\psi \rightarrow\left|\psi_{\mathrm{c}}\right|$ as $R \rightarrow \infty$. It means that no breaking is possible at the wave front.

\section{Conclusions}

It has been shown that an unsteady radiation field gives rise to a radiation induced wave. It is found that the nonlinearity in the governing differential equations contributes nothing towards radiation induced waves, these waves ultimately are damped. We have seen that in the case of plane wave front when $Q_{0}>0$, a shock formation is either delayed or disallowed due to thermal radiation and magnetic field effects. On the other hand, thermal conduction has a destabilizing effect on the weak wave propagation because it accelerates the process of termination of a weak wave in a shock wave. When $Q_{0}<0$, the thermal radiation and magnetic field with finite electrical conductivity decrease the time of breaking of weak waves, while thermal conduction increases the tine of breaking of weak waves in shock waves. In the case of cylindrical wave front an increase in $Q_{0}$ or an increase in the initial curvature will increase the time of breaking of the wave front, while for $Q_{0}<0$ no breaking is possible at the wave front.

\section{References}

[1] T.Y. Thomas, J. Malh. Mech. 6, 453 (1957).

[2] C.N. Gaul, J. Malh. Mech. 10, 393 (1961).

[3] G.A. Nariboli, J. Math. Mech. 12, 141 (1963).

[4] D.Y. Ludwig, A.E.C. Report 1961, NYO-9351, New York.

[5] G.F.D. Duff, Cummun. Pure Appl. Math. 1, 189 (1964).

[6] G.A. Nariboli, B.G. Secrest, Tensor (N.S.) 18, 22 (1967).

[7] J.B. Helliwell, J. Fluid Mech. 37, 497 (1969).

[8] J.B. Helliwell, M.F. Mosa, Int. J. IIcat Mass Transf. 22, 657 (1979).

[9] W.G. Vincenti, B.S. Baldwin, J. Fluid Mech. 12, 449 (1962).

[10] V.A. Nye, Q. J. Mech. Appl. Math. 23, 247 (1970).

[11] S. Srinivasan, R. Ram, Z. Angew. Malh. Phys. 26, 307 (1975).

[12] R. Ram, Appl. Sci. Res. 34, 93 (1978).

[13] R. Ram, B.D. Pandey, Indian J. Pure Appl. Math. 10, 950 (1979).

[14] R. Shankar, M. Prasad, Z. Angew. Malh. Phys. 30, 937 (1979).

[15] A.S. Rai, M. Gaur, Acla Phys. Pol. A57, 653 (1980).

[16] W.G. Vincenti, C.H. Kruger, Introduction to Physical Gasdynamics, Wiley, New York 1977, p. 491.

[17] T.Y. Thomas, J. Malh. Mech. 6, 311 (1957).

[18] E.P. Lane, Metric Differential Geometry of Curves and Surfaces, Chicago Univ. Press, Chicago 1940, p. 209. 